\title{
Vers une politique publique de l'archivage audiovisuel ? Le cas vaudois
}

\section{Raphaël Berthoud}

\section{Introduction}

Depuis plus d'un siècle les images animées font partie de notre paysage culturel ; il est aujourd'hui évident qu'elles jouent un rôle important comme mémoire vivante, témoins privilégiés d'un contexte et d'une époque. Au même titre que nous nous sommes préoccupés jusqu'ici des documents écrits, le moment n'est-il pas venu de nous préoccuper aujourd'hui de l'« image animée », soit des documents audiovisuels?

Pour ce pan de mémoire, les politiques de conservation tardent à s'exprimer. Ce retard est lié à la conservation problématique des supports : la volumétrie, les équipements nécessaires à leur lecture, le respect des normes de description et de conservation - souvent récentes et contraignantes sur le plan juridique - démotivent les institutions. A cela s'ajoutent la variété des supports, l'obsolescence de ceux-ci, la qualité relative des supports de substitution (CD, DVD), mais aussi toutes les questions concernant leur restauration.

Avant de continuer, il est nécessaire de se demander qui a les compétences techniques, intellectuelles et professionnelles pour évaluer aujourd'hui ce patrimoine audiovisuel. Il semble que l'archiviste, de par ses qualifications pointues en archivistique et en histoire, soit à même d'assumer le rôle d'expert évaluateur, afin d'analyser avec pertinence non seulement les supports en tant que tels et les problèmes techniques qu'ils posent, mais également de définir et de décrire les émetteurs, les récepteurs, les diffuseurs et les acteurs de ces données particulières.

Ces dernières années, cette thématique a été abordée à quelques reprises ; Frédéric Sardet, chef de service Bibliothèques \& Archives de la Ville de Lausanne, s'inquiète dans un article intitulé «Sommes-nous en train de créer des cimetières? " d'avril $2005^{1}$ ou encore l'ancien directeur de l'Institut national français de l'audiovisuel Emmanuel Hoog, Une mémoire audiovisuelle qui s'estompe en silence du 3 octobre $2005^{2}$. Le souci de sauvegarder la mémoire audiovisuelle ressort clairement aussi au niveau des médias. La radio et la presse se sont saisis également de cette thématique (voir l'article, Le patrimoine télévisuel en danger paru dans Le Temps du

Sardet, Frédéric, Sommes-nous en train de créer des cimetières ? in Arbido, avril 2005, pp. 12-15. Hoog, Emmanuel, Une mémoire audiovisuelle qui s'estompe en silence in Le Devoir, 3 octobre 2005, https://www.ledevoir.com/opinion/idees/91750/une-memoire-audiovisuelle-qui-s-estompe-en-silence, le 10 juin 2019. 
10 janvier 2004, signé par Didier Pradervand ${ }^{3}$ ou plus récemment l'émission de radio RTS1 : « On nous dit rien », du 18 octobre 2017, « Les archives audiovisuelles, un patrimoine en péril », signé de Renaud $\mathrm{Malik}^{4}$ ). Ce souci est également porté par l'Association des archivistes suisses qui a proposé à ses membres, en août et septembre 2018, une formation spécifique intitulée : «Archiver des documents audiovisuels, théorie et pratique ».

Non seulement les professionnels des archives et les médias s'inquiètent de la conservation de l'audiovisuel, mais également les politiciens font part de leurs soucis et demandent des explications pour connaître la politique de conservation et de prise en charge de ce patrimoine. Le $1^{\text {er }}$ septembre 2015, la députée vaudoise Fabienne Freymond Cantone dépose un postulat, co-signé par 23 députés, auprès du Conseil d'État vaudois intitulé «Les Archives cantonales vaudoises, mémoire de notre canton, quid des archives audiovisuelles ? ».5

Ce postulat vaudois a motivé notre réflexion pour l'élaboration de ce travail de master. L'objectif est de mettre en résonnance les problèmes posés par la conservation de ces documents audiovisuels avec les besoins et les exigences du travail de l'archiviste d'une part, mais aussi avec les ressources tant financières qu'humaines disponibles dans le Canton de Vaud, d'autre part.

Dans le cadre de cet article, nous commençons par définir brièvement le concept de l'audiovisuel à proprement parler et ses différentes acceptions. Nous passerons rapidement sur les difficultés que posent les supports et les formats que l'on trouve dans archives audiovisuelles. Nous abordons ensuite la production audiovisuelle vaudoise dans le cadre des institutions cantonales en laissant de côté les archives audiovisuelles privées, puisque les exigences posées pour cette publication ne s'y prêtent guère. Le chapitre suivant s'attache à décrire la situation actuelle aux Archives cantonales vaudoises. Pour terminer, nous proposons des solutions et des scénarios politiquement et financièrement envisageables pour prendre en charge ce patrimoine.

\section{Prolégomènes}

Dans ce travail, nous nous appuyons sur les définitions successives données par l'Organisation des Nations Unies pour l'éducation, la science et la culture (UNESCO) en 1974 dans sa résolution 3422, préoccupation appuyée par une résolution demandant de préserver les images des pays en voie de développement en 1979, par la

3 https://www.letemps.ch/culture/patrimoine-televisuel-danger, le 24 juin 2018.

4 https://www.rts.ch/play/radio/la-matinale/audio/on-nous-dit-rien-les-archives-audiovisuelles-unpatrimoine-en-peril?id=8990230, le 24 juin 2018.

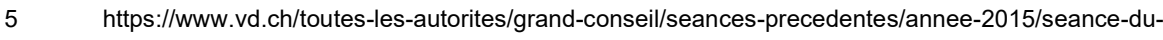
mardi-1er-septembre-2015/postulat-fabienne-freymond-cantone-et-consorts-les-archivescantonales-vaudoises-memoire-de-notre-canton-quid-des-archives-audiovisuelles/, le 10 juin 2019 
recommandation de 1980 à Belgrade, puis par des recommandations pratiques, en 1985, plus précisément sur la définition du groupe de travail Audiovisual Archiving Philosophy Interest Network (AVAPIN) et l'UNESCO, sous la plume de Ray Edmondson $^{6}$ :

3.3.2.4 Constituent des documents audiovisuels les ouvres comprenant des images et/ou des sons reproductibles réunis sur un support matériel dont :

- l'enregistrement, la transmission, la perception et la compréhension exigent le recours à un dispositif technique ;

- le contenu visuel présente une durée linéaire

- le but est de communiquer ce contenu et non d'utiliser la technique mise en ouvre à d'autres fins.

En tant qu'européen, nous prendrons donc une définition très restrictive : les supports non textuels, comme la photographie (diapositives y compris) et les microfilms qui ont aussi besoin d'un appareil technique «médiateur» ne seront pas considérés comme des sources audiovisuelles dans cette étude. Il faut bien rendre attentif au fait que cela laisse de côté les diaporamas (diapositives accompagnées d'une bande son, qui déclenchait par un procédé électrique le passage à la diapositive suivante), procédé très en vogue dans les années 1970-1980, encore présent dans certains musées (Musée du Gruyère à Gruyères, les Grottes de Vallorbe, les Mines de Sel de Bex, avant la refonte de l'exposition en 2018).

Nous éliminerons de ce travail de master (faute de place) le cinéma et la radio, qui font partie du domaine audiovisuel mais qui tous deux ont la chance d'occuper une place de choix avec leurs deux institutions dédiées : la Cinémathèque suisse et la Phonothèque nationale suisse (de création récente toutefois, 1987).

Nous conserverons donc l'audiovisuel dans le sens images en mouvement, non publiées, à utilisation non commerciale, quel que soit leur support et leur accompagnement sonore. De plus, nous ajouterons la limitation d'un cadre géographique restreint : le canton de Vaud.

\section{Archives audiovisuelles : valeurs patrimoniales et historiques}

Comme le définit l'UNESCO dans sa recommandation de Belgrade de 1980, les images en mouvement font partie de la culture patrimoniale des pays et doivent à ce titre être préservées ${ }^{7}$ :

Considérant que les images en mouvement sont une expression de l'identité culturelle des peuples et qu'en raison de leur valeur éducative, culturelle,

6 Edmondson, Ray, Audiovisual archiving, philosophy and principles, United Nations Educational, Scientific and Cultural Organization, Paris, 20163

7 https://unesdoc.unesco.org/ark:/48223/pf0000114029_fre, le 10 juin 2019 
artistique, scientifique et historique, elles font partie intégrante du patrimoine culturel d'une nation, [...]

Considérant que les images en mouvement sont aussi un moyen fondamental d'enregistrer les événements à mesure qu'ils se déroulent et qu'à ce titre elles constituent, de par la nouvelle dimension qu'elles apportent, des témoignages importants et souvent irremplaçables de l'histoire, du mode de vie et de la culture des peuples ainsi que de l'évolution de l'univers, [...]

Les mesures appropriées devraient être prises pour veiller à ce que le patrimoine d'images en mouvement bénéficie d'une protection matérielle satisfaisante contre les atteintes du temps et de l'environnement.

Il ne s'agit pas tant dans cette réflexion de sauvegarder la programmation de ces médias (bien assez illustrés par les sources écrites) que de protéger leurs produits à des fins historiques et/ou sociologiques de notre civilisation pour les générations futures. Qui peut savoir actuellement où iront leurs intérêts?

\section{Types des archives audiovisuelles}

\section{Support et format}

Ces deux éléments présentent à eux seuls la grande difficulté du traitement des archives. L'obsolescence et les techniques (parfois totalement propriétaires d'une marque) sont un rempart au traitement archivistique de ces données. Pourtant un archiviste ne peut se contenter de mettre de côté, voire pire, éliminer des sources parce qu'illisibles.

\section{Conservation}

La conservation est la partie la plus simple. Nous connaissons par expérience de pays étrangers, la manière de conserver les supports originaux. MEMORIAV a édicté aussi des recommandations à ce sujet ${ }^{8}$. Sur le plan audiovisuel tel que nous l'entendons, une fois le contenu lu et conservé sur un support numérique - selon les normes archivistiques-, nous pensons que nous avons le droit d'éliminer le support cassette vidéo. A quoi bon conserver un élément dont on sait que la technique de lecture disparaît et dont le support matériel lui-même a une durée de vie très limitée ? Les cassettes portent elles-mêmes des contenus dont il est facile de dire qu'ils sont souvent des copies d'un original disparu. 


\section{Restauration}

La restauration est un élément technique visant à pallier les lacunes, les éléments abîmés. Cette technique fait appel à la notion de transformation. Cette opération est un gouffre financier. Peut-être qu'il vaut mieux avoir un écran noir (perte d'informations) à un moment précis : c'est l'état dans lequel l'archiviste a reçu l'élément audiovisuel.

\section{Numérisation}

On ne pourrait parler de conservation sans passer par cette étape de numérisation. Cette manière de faire déplace la notion d'original : ce n'est plus le support cassette mais le contenu qui devient « original ». La rapidité avec laquelle les techniques changent pousse à trouver l'élément le plus stable qui est, actuellement, la numérisation. Toutefois, cette technique tend à muter de manière continuelle. Il y a donc un besoin de s'appuyer sur des standards libres de droits. Cela demande un suivi constant. On peut imaginer deux standards différents : l'un sans compression, pour la conservation, et une copie compressée pour la diffusion (internet ou salle de lecture).

De plus s'ajoute à ce problème de standards une réflexion sur les éléments pouvant contenir la masse de téraoctets exigée par une telle opération. Doit-on les conserver sur CD ou DVD, dont les supports, on le sait maintenant, sont loin d'être pérennes? Ou alors, comme cela se fait en France, sur des cassettes Linear TapeOpen, qui contiennent de grandes capacités mémoire ? Là aussi l'obsolescence de tels supports peut faire craindre le pire. A notre avis, les supports pour l'instant les plus fiables sont des serveurs mais dont la quantité (et donc le prix) pourrait effrayer n'importe quelle direction informatique.

\section{Inventaire - catalogage - métadonnées}

L'inventaire est l'élément le plus important. L'inscription des métadonnées et le suivi des normes ISAD(G), ISAAR par un archiviste sont primordiaux. Sur cette colonne vertébrale constituée de l'inventaire, un chercheur doit pouvoir trouver l'élément cherché, quelle que soit la localisation du support, s'il n'est pas conservé sur le lieu même des archives. Toutes les normes archivistiques en vigueur permettent d'inventorier ou de cataloguer les sources audiovisuelles (ISAD (G), ISAAR, Dublin Core, METS, SEPIADES)

Le sujet, les personnes intervenantes si possible, la date et le lieu de tournage font partie des métadonnées de base, sans quoi l'absence de contexte rend difficile l'interprétation des sources. Doivent s'ajouter à cela des métadonnées du support (format de l'image, qualité de numérisation, standard de filmage) et éventuellement, si les éléments n'ont pas été formatés aux standards d'archivage, au moins le format de 
codage et les codecs ${ }^{9}$ utilisés. Il est important toutefois de souligner ici la nécessité absolue de traiter les fonds d'archives audiovisuelles comme n'importe quel fonds papier : le respect de l'unité du fonds, de la provenance et de l'origine est l'élément essentiel.

\section{Indexation}

Une indexation doit pouvoir se faire, par éléments descripteurs. Deux indexations sont possibles : la première par « bobine » ou par cassette, indispensable pour tout inventaire cohérent et scientifique : Qui (a réalisé/produit) ? De quoi parle-t-on (thème ou sujet) ? Qui parle ? Quand ? Et surtout, quelle technique a-t-elle été utilisée ? Voire à quel endroit la séquence a-t-elle été tournée ? La seconde consiste à créer une indexation très poussée (découpage thématique par segments) dont un exemple en Suisse a été réalisé par l'Association Plans fixes (http://www.plansfixes.ch/indexation/) : elle permet d'effectuer des recherches par mots-clés contrôlés ou en texte libre. Nous constatons toutefois par la liste des mécènes (Loterie romande, Swisslos Berne, Fondation Sandoz, MEMORIAV, Cantons du Jura, de Fribourg, du Valais et de Vaud, Ville de Genève) avec la collaboration de l'Université et l'École polytechnique de Lausanne, à quel point cette indexation manuelle complète est gourmande en ressources financières et humaines. On peut imaginer que la technique de la reconnaissance informatique automatisée pourra permettre dans un deuxième temps une indexation rapide et approfondie, alors que le Canton de Vaud pourrait trouver des aides techniques, avec la présence de grandes institutions techniques que sont l'Université de Lausanne, l'École polytechnique fédérale de Lausanne, la Haute école d'Ingénierie et de Gestion, basée à Yverdon. Certains informaticiens des trois écoles pourraient être intéressés au développement d'un logiciel permettant une indexation informatique des données contenues sur des documents audiovisuels.

\section{Communication}

On ne peut parler d'inventaire et de transformation par la numérisation des documents audiovisuels sans penser à terme la diffusion et la communication. Sur ce plan, de nombreux points sont encore flous qu'il faut absolument traiter de prime abord : les droits d'auteur, les droits de communication, le droit à l'oubli. Il n'en reste pas moins que travailler les points précédents sans la mise à disposition des sources audiovisuelles et sans la prise en compte du désir légitime de l'usager de pouvoir accéder à cette documentation rend le travail vain. MEMORIAV soumet d'ailleurs sa participation à l'ouverture des archives audiovisuelles. Le nombre de personnes demandant

$9 \quad$ Un codec est un dispositif matériel ou logiciel permettant de mettre en œuvre l'encodage ou le décodage d'un flux de données numérique, en vue d'une transmission ou d'un stockage. Certains codec intègrent également une fonction de compression ou encore de chiffrement des données. 
des archives de la télévision française à l'INA et la masse des demandes sur internet de personnes cherchant des émissions de la RTS en sont une preuve suffisante pour comprendre l'intérêt de la communication et de la diffusion des sources audiovisuelles.

\section{La plus-value de l'authenticité garantie par l'art archivistique}

Une politique d'archivage est nécessairement fondée sur une politique de versements (lorsqu'il s'agit d'archives officielles) ou sur une politique d'acquisition (dans le cas d'archives d'origine privée). Elle permet d'afficher les objectifs poursuivis, les critères d'évaluation et l'intégration de l'institution dans l'environnement des institutions patrimoniales.

Par le traitement scientifique des archives audiovisuelles, par l'inscription de métadonnées (automatique ou manuelle), l'archiviste garantit l'authenticité des documents audiovisuels. Il peut aussi définir le statut de ces documents : visée informative, visée publicitaire, visée persuasive, voire visée ludique. De plus, par ses connaissances scientifiques, et une formation continue, l'archiviste est à même de donner un calendrier de conservation aux organismes versants, ainsi que les formats d'archivage nécessaires, et de définir (éventuellement) la valeur archivistique de certains documents en collaboration avec l'organisme verseur ou donateur.

\section{Production vaudoise}

En déposant son postulat dont nous avons déjà parlé, la députée Fabienne Freymond Cantone pose la question de la sauvegarde du patrimoine vaudois audiovisuel. En liant clairement le rôle des Archives cantonales à la réflexion sur les documents audiovisuels, elle dresse déjà un portrait inquiétant :

Se pose ainsi la question de la conservation des films et enregistrements des séances du Grand Conseil. A ce jour, les ACV n'ont pas de structure technique pour le faire. Des accords sont passés avec les Archives de la Ville de Lausanne et la Cinémathèque suisse pour l'exploitation de ces sources. Cependant, au nom de leur conservation, il serait logique que, selon qu'ils soient sonores ou filmés, ces compléments aux procès-verbaux de séances du Grand Conseil soient conservés avec ces derniers et pas disséminés dans d'autres institutions.

L'attentisme et l'absence de position claire actuels vont provoquer des coûts exorbitants. La mémoire cantonale est en péril non seulement en raison de sa diversité et de sa masse qui crô̂t de manière exponentielle, mais surtout en raison des contraintes liées à ses nouveaux supports, évoluant sans cesse en fonction de la technologie. Forts de ces différentes observations et réflexions, 
nous avons l'honneur de demander au Conseil d'État, par la voie de ce postulat, de faire un état des lieux sur la question de l'archivage des documents audiovisuels. De plus, une position de nos Autorités sur l'avenir à donner aux archives audiovisuelles qui témoignent du présent et de l'histoire de notre canton - autant celles institutionnelles que celles d'intérêt pour la mémoire collective - devient nécessaire, cela tant pour leur conservation, leur valorisation, que pour leur diffusion.

A notre connaissance, la production vaudoise audiovisuelle se divise en deux parties, dont une - la partie réalisée et tournée par des prestataires privés (télévisions, personnes particulières) - sera mise de côté dans cet article:

\section{Institutionnelle, services de l'Etat}

Nous nous appuyons ici sur les informations données par Gilbert Coutaz, directeur des Archives cantonales vaudoises, qui a mené une enquête sur la production audiovisuelle des différents services de l'administration. Sous la double signature du Chancelier d'État et du directeur des ACV, 42 questionnaires accompagnés d'une lettre explicative ont été adressés, le 6 mars 2017, à l'ensemble des chefs services de l'administration cantonale et de l'Ordre judiciaire, ainsi qu'au Secrétaire général du Grand Conseil. Ce questionnaire dresse un portrait exhaustif de la production audiovisuelle étatique.

La progression de la volumétrie se constate dans ce pré-inventaire, par le recours de plus en plus courant à des clips de présentation des différents services, certains secteurs recourant au reportage cinématographique et aux interviews pour présenter certains types d'engagement ou d'événements particuliers. Certaines filières de formation (surtout le Service de l'enseignement post-obligatoire) et la télévision interne des Établissements de la Plaine de l'Orbe recourent elles aussi à des productions audiovisuelles.

De manière générale, les archives audiovisuelles concernent peu de secteurs mais les quatre plus gros producteurs d'archives audiovisuelles sont : la Police cantonale ; le Centre d'Enseignement Médical et de Communication Audiovisuelle (CEMCAV) de l'Hôpital Cantonal et Universitaire, devenu le Service d'Appui Multimédia (SAM) le 1er juillet 2016 ; le Centre de formation professionnelle forestière et le Grand Conseil.

Une remarque s'impose sur cette dernière production: les séances du Grand Conseil avant 2010 ont été copiées de VHS sur DVD ; depuis le 24 août 2010, les séances sont disponibles sur le site du responsable des installations techniques de la salle du Grand Conseil, un prestataire privé (Sonomix) et sauvegardées sur des serveurs externes, avec les problèmes de sécurité que cela pose. Plusieurs copies de fichiers sonores sont sécurisées sur un disque dédié sur les serveurs de l'État de Vaud. 
Bien que la valeur probatoire légale des séances du Grand Conseil vaudois ait été attribuée à l'écrit (après corrections des divers intervenants), une séance du Grand Conseil filmée permet de « ressentir » l'ambiance d'une séance, et de ce fait de compléter l'écrit. Si l'audiovisuel était amené à ne pas être conservé, ne passerait-on pas à côté d'expressions qui permettraient de comprendre davantage les échanges entre les interlocuteurs du Grand Conseil ?

Nous choisirons ici deux exemples de productions institutionnelles, le premier qui a longtemps préoccupé Gilbert Coutaz, le CEMCAV et le second, arrivé durant la rédaction de ce master, la demande de l'ERACOM (l'École Romande d'Arts et de Communication) située à Lausanne.

\section{Le CEMCAV (=SAM)}

Créé en 1974, afin de doter le Centre hospitalier vaudois de moyens d'enseignement, d'une bibliothèque et d'un centre de documentation, le CEMCAV a réalisé une enquête en 2004 : il comptait près de 25'000 travaux différents que ce soit sous forme de photographies, de graphisme, de vidéos et de multimédia.

En totalité, près de soixante films en $16 \mathrm{~mm}$, dix-sept émissions sur la santé, diffusées jusqu'en 2003 par la Télévision de la région lausannoise, mille heures de cassettes, dont le transfert sur support numérique représente cinquante téraoctets nous mettons à dessein de côté les photographies. Toutefois, du matériel de lecture a été conservé afin de pouvoir lire ces anciens supports. La direction du CHUV met à disposition une personne pendant deux jours par semaine, encadrée par les Archives cantonales, afin de créer l'inventaire exact. Différents films tournés à l'hôpital de Cery entre 1962 et 1982 ont été confiés aux soins de la Cinémathèque suisse. Cette production est toutefois, pour des questions de respect de fonds, inventoriée dans l'inventaire des Archives cantonales sous les cotes SB $258 \mathrm{C} / 1$. Actuellement le CEMCAV gère cette politique de numérisation et de sauvegarde des documents audiovisuels, avec l'appui des Archives cantonales vaudoises.

\section{L'ERACOM}

Fondée à Lausanne en 1942 sous le nom d'École romande de typographie, nom qu'elle portera jusqu'en 1971, elle se spécialise dès 1972 dans les arts graphiques (sous l'acronyme ERAG jusqu'en 1999). Elle devient cantonale en 1992, selon la loi vaudoise sur la formation professionnelle ${ }^{10}$. Elle changera de nom durant l'année 2000 pour devenir l'ERACOM avec l'ajout de la formation de concepteurs en multimédia.

Nous avons eu le privilège dans le cadre de ce travail de master de pouvoir assister à un entretien entre le directeur des Archives cantonales vaudoises et le 
directeur de l'ERACOM, accompagné de deux responsables informatiques, l'un de l'École et l'autre du Service informatique de la Direction Générale de l'Enseignement post-obligatoire, tous préoccupés par la sauvegarde de leurs documents, en particulier audiovisuels. La destination tant artistique qu'en communication de cette École permet de comprendre la volumétrie des documents de ce type dans cette organisation, et peut-être de définir les difficultés que cela provoque, si le soutien de la Direction des services informatiques de l'État de Vaud n'est pas acquis.

Au moment de l'entretien, soit le 27 avril 2018, nous avons appris que chaque élève doit, dans le cadre de ses études, établir une production audiovisuelle ou graphique - parfois dans le cadre de son diplôme de fin d'année. Actuellement, par manque de ressources sur le serveur dédié à cette École par le Canton, les élèves et les enseignants travaillent avec des disques durs plug and play, sans sauvegarde. Ils conservent ces disques durs durant toute la durée de leurs études. A la remise du travail de diplôme, l'ERACOM conserve durant une année le travail de l'élève, le disque dur est ensuite reformaté pour être transmis à un autre élève.

Cette manière de faire révèle l'absence de calendrier de conservation, ainsi que celle de critères objectifs permettant d'expliquer la valeur des travaux ou du moins de justifier la production d'une école âgée de septante-cinq ans.

Les premiers documents audiovisuels datent des années 1970. Les élèves travaillent depuis environ une dizaine d'années en numérique, avec des caméras $4 \mathrm{~K}$. Cela signifie par exemple pour un travail de 25 heures de tournage (ou deux jours de tournage) une production de vingt téraoctets pour un seul travail. La volumétrie des documents de l'École purement audiovisuels sur les 3 ans d'études des élèves correspondant à des dizaines de péta-octets ( $10^{15}$ octets). L'ERACOM ne possède que le simple enregistrement sur le réseau cantonal pour conserver certains travaux et « doit mendier chaque téraoctet » auprès de la Direction des Services Informatiques de l'État de Vaud (DSI), dépendant du Département des infrastructures et des relations humaines (DIRH).

Quand nous apprenons que l'État de Vaud a prévu dans le cadre de son exposé des Motifs pour le Records Management environ 300 téraoctets ( $10^{12}$ octets) et que la direction des services informatiques conservait, en 2010, 500 téraoctets de documents numériques, la différence entre le besoin de capacité de sauvegarde de cette école et la totalité de capacité de sauvegarde du Canton, est énorme.

Il faut rapidement définir l'urgence de la conservation. Selon le directeur de 1 'ERACOM, il est indispensable de conserver la production de l'École en particulier celle qui a été primée lors de festivals internationaux ou nationaux. Une définition stricte des standards d'archivage et des codecs ${ }^{9}$ doit se faire de toute urgence, car les responsables informatiques se trouvent devant plus de mille codecs possibles, qui sont parfois propriétés d'une firme, très souvent incompatibles avec certaines plates- 
formes, même en interne. Ce travail de réflexion doit se faire rapidement, car l'ERACOM a obtenu le mandat, avec ses étudiants, de créer l'identité des Jeux Olympiques de la Jeunesse en 2020 qui auront lieu à Lausanne par des clips, des reportages et des bandes annonces. Le tournage de certaines séquences a déjà commencé : la pose de la première pierre du Vortex, bâtiment destiné à accueillir les délégations sportives puis à devenir un campus d'étudiants.

Comme nous l'avons défini dans la partie 2 de notre master, ces documents font forcément partie du patrimoine culturel du canton de Vaud.

\section{Les Archives cantonales : situation actuelle}

Le postulat de la députée Freymond Cantone pose la question du positionnement des Archives cantonales (et non d'une autre institution).

La mission des Archives cantonales vaudoises est traditionnellement liée à la conservation de l'écrit, et elles ne souhaitent pas être l'institution cantonale de référence en la matière, comme le dit Gilbert Coutaz dans son rapport d'activités 2003 :

Les Archives cantonales vaudoises ne sont pas et ne seront jamais l'institution cantonale de référence pour les archives photographiques, sonores et filmiques. Elles doivent plutôt rechercher des formules de partenariat avec des institutions patrimoniales du canton, là où des archives photographiques, sonores et filmiques sont mêlées à des documents écrits (= fonds mixtes). Dans les cas de fonds comprenant des supports d'informations variés, elles veillent à sauvegarder l'unité des fonds ; s'il s'agit de fonds composés exclusivement de photographies, de témoignages sonores, de films, elles conseillent leur remise aux institutions spécialisées, en particulier au Musée de l'Élysée, aux Archives de la Ville de Lausanne et à la Cinémathèque suisse. ${ }^{11}$

Dans la réalité, les Archives cantonales vaudoises se trouvent tout de même confrontées à deux types d'archives audiovisuelles. Ces documents reçoivent la cote de gestion U s'ils sont analogiques ou W s'ils sont numériques. Un rapport dressé par une stagiaire de la Haute Ecole de Gestion de Genève, Lea Ritter, encore en cours d'écriture au moment de la rédaction de ce master, analyse l'état de conservation de l'existant (cote de gestion U image animée analogique) et sera remis au Directeur des Archives cantonales vaudoises. Elle ne dénombre pas moins de 9 formats analogiques différents : près de 380 films de dimensions diverses et 470 cassettes VHS. Il y a encore dans la cote $\mathrm{W}$ (format numérique) près de $270 \mathrm{CD}$ ou DVD. ${ }^{12}$ 


\section{Les archives audiovisuelles institutionnelles}

Selon la loi sur l'archivage du canton de Vaud (article 9, alinéa 1) de 2011 :

${ }^{1}$ Les Archives cantonales vaudoises veillent à la constitution des archives historiques des autorités cantonales, assurent leur conservation et facilitent leur consultation. [...]

et selon l'article 3 , alinéa 1 :

${ }^{1}$ On entend dans la présente loi par :

document : toutes les informations, enregistrées sur quelque support que ce soit, en particulier sur support électronique, produites ou reçues par les autorités mentionnées à l'article 2, ainsi que tous les instruments de recherche et toutes les données complémentaires qui sont nécessaires au repérage, à la compréhension et à l'utilisation de ces informations;

Selon ces deux articles, il est évident que toute production de l'État doit être évaluée et conservée par les Archives cantonales, quel que soit leur support. Comme nous l'avons précédemment présenté, les organismes de l'État produisent des documents audiovisuels, qui ont légalement leur place aux Archives cantonales vaudoises. Il n'y a pas ici nécessité d'établir s'il faut conserver ces documents ; cela est compris dans le périmètre de la loi.

Toute production audiovisuelle étatique a forcément laissé des traces écrites (contrats, discussions, échanges épistolaires ou mails), ce qui conforte la directive établie. Néanmoins, nous ne pouvons que constater que la volumétrie des documents qui vont être versés aux Archives cantonales est très importante et l'absence d'un calendrier de conservation clair et précis pour les documents audiovisuels n'aide pas, contrairement à ce qui est défini pour les archives papier ; cela est certainement dû à la nouveauté des supports. La rétro-numérisation, liée à l'obsolescence des moyens techniques et à la qualité médiocre des supports utilisés nécessite un important apport financier auquel l'administration devra faire face. Actuellement, faute de moyens techniques, de ressources financières et de ressources en personnel spécialisé, ces documents audiovisuels sont conservés dans des lieux de stockage adaptés à de tels supports, sauf quand il s'agit de documents numériques encore en cours d'utilisation (archives intermédiaires). Les Archives cantonales ont pour tâche de guider les institutions étatiques dans l'établissement de leur calendrier de conservation et sur la hiérarchisation de la valeur archivistique des documents.

Si nous reprenons l'exemple de l'ERACOM, la durée de conservation des travaux d'élèves (animés, en 3D, en haute qualité) est actuellement d'une année, en cas

conservés tous les supports CD-DVD, qu'ils contiennent des textes, des photographies, des images animées, parce que les Archives cantonales ne possèdent pas les moyens techniques (lecteurs) d'analyser le contenu de ces supports. Parfois sous la cote de gestion $U$ (film analogique) se trouvent aussi des éléments numériques qui auraient dû être placés sous la cote de gestion W (numérique) en cours de corrections, après l'écriture de ce master. 
de recours de la part d'un étudiant. Il est toutefois nécessaire de conserver des travaux que l'ERACOM juge particulièrement intéressants, que ce soit par la technique utilisée, par le sujet présenté (l'habillage graphique des Jeux olympiques de la Jeunesse), ou par l'obtention de prix lors de concours internationaux, nationaux et cantonaux, travaux qui légitiment l'existence de cette institution interne à l'État. Comment savoir si parmi les élèves de l'ERACOM qui recevront leur titre en 2018, il n'y en aura pas quelques-uns qui, plus tard, recevront un prix ou seront particulièrement mis en avant? Il serait intéressant que l'ERACOM puisse montrer au-delà d'une année la production de ses élèves remarqués plus tardivement dans leur carrière.

\section{Le calendrier de conservation}

Il est important de dialoguer et de collaborer avec les organismes versants. Avec la mise en place d'une gestion électronique des documents au sein de l'État de Vaud et une livraison sous format informatique des données, les Archives peuvent imposer la mise en place d'un calendrier de conservation et définir surtout les formats dans lesquels les documents doivent être versés.

\section{La valeur archivistique de la production institutionnelle}

Elle est particulièrement difficile à définir. Que vaut-il la peine de conserver ? Une bonne conservation est aussi la résultante d'une bonne élimination. Pourtant, dans le cadre de la production institutionnelle, notre réponse à cette question sera curieusement orientée vers la notion du « plus possible ». En effet, chaque image produite dans le cadre institutionnel a été réfléchie, puisque chaque franc investi vient du contribuable : l'administration doit pouvoir prouver l'existence du travail demandé. Certaines images qui paraissaient peut-être obsolètes seront une source d'information immense pour le chercheur, voire pour le grand public. Nous prendrons par exemple les images du Service de l'aménagement territoire (il s'agit là de photographies aériennes). Le développement du canton, les infrastructures créées sont visibles sur les images créées.

Nous pensons donc que la valeur archivistique de toute production audiovisuelle institutionnelle est grande et mérite, suivant en cela la loi sur l'archivage, une considération particulière.

\section{Retour sur la situation actuelle des Archives cantonales vaudoises}

Actuellement dans le canton de Vaud, aucun travail de rétro-numérisation n'est prévu, à part les collaborations entamées avec la Cinémathèque suisse pour les archives du 
Cineac ${ }^{13}$, la Radio télévision suisse romande pour le Fonds Jack Rollan. (PP 881/556583 ) et les Archives communales de Lausanne pour le Fonds du Réarmement moral de Caux (PP 746/9.6).

La direction des Services Informatiques (DSI) ne met pas de serveurs en place autres que ceux qui sont prévus pour la bonne marche quotidienne de l'État. Comme nous l'avons déjà dit, la sauvegarde des documents audiovisuels nés numériques est donc aussi en péril. Les réponses ponctuelles trouvées par les ACV sont une manière de réagir aux défis techniques que nous lance l'audiovisuel, par exemple en décidant de transférer auprès de la Cinémathèque suisse les archives filmiques conservées par les Archives cantonales, en dehors des supports numériques. Dans cette démarche, si l'idée de conservation est bien présente, la valorisation des informations est omise. La question des supports obsolètes (VHS, Betacam, U-Matic) reste ouverte. Le Canton de Vaud - du moins ses autorités politiques - a manqué d'une vision d'avenir en laissant partir les appareils techniques de lecture des sources audiovisuelles qui étaient à disposition à Territet entre 1983 et 2015. Les appareils du Musée national suisse de l'audiovisuel (AUDIORAMA) se trouvent maintenant incorporés au musée ENTEROnline à Soleure. Les archives écrites de cette Fondation ont été déposées pourtant aux Archives cantonales vaudoises. ${ }^{14}$

La mutation du paradigme social ${ }^{15}$ exige aussi un changement de paradigme de conservation. Les Archives cantonales doivent donc viser l'accroissement des documents audiovisuels pour constituer la mémoire cantonale patrimoniale et historique.

\section{Un institut vaudois de l'audiovisuel : une utopie ?}

Le terme « audiovisuel » consiste non seulement dans une définition des supports, des formats, et du contenu mais encore une dimension institutionnelle, selon le rapporteur de l'UNESCO, Ray Edmondson :

3.3.3.2 Les archives audiovisuelles sont un établissement ou un service d'un établissement qui a pour mission statutaire ou autre de mettre à disposition un fonds de documents audiovisuels et le patrimoine audiovisuel en en assurant la collecte, la gestion, la conservation et la promotion. Lausanne dans les années 1936 à 1968. La Télévision Suisse Romande a acquis une partie de ce fonds peu documenté. De nombreux sujets étaient déjà tournés sur de la pellicule couleur, cela dès les années 1940 (https://www.rts.ch/archives/tv/culture/notre-passe-a-vif/6833282-le-cineac-.html), le 8 septembre 2019

14 http://www.audiorama.ch, le 28 mai 2018.

15 Dans les sciences sociales, le terme est employé pour décrire l'ensemble d'expériences, de croyances et de valeurs qui influencent la façon dont un individu perçoit la réalité et réagit à cette perception. Ce système de représentation lui permet de définir l'environnement, de communiquer à propos de cet environnement, voire d'essayer de le comprendre ou de le prévoir. 
Dans le monde archivistique, deux manières de faire se présentent : le dépôt légal (en France, au Québec, au Maroc, et au Sénégal) ou la donation (Allemagne).

Pour éviter toute perte, un dépôt légal pourrait s'imposer. Peut-on créer un institut national de l'audiovisuel ou par défaut un institut cantonal ? En 1992, la Confédération a répondu négativement à l'idée de créer un centre national d'information audiovisuelle à cause des coûts trop élevés (cause alléguée). La cause réelle en est certainement que le fédéralisme aurait sans doute pâti d'un modèle centralisateur, chacune des parties linguistiques de la Suisse pouvant revendiquer une exception culturelle et donc une part financière importante. La naissance de l'association MEMORIAV pallie cet état de fait : elle œuvre de manière active et durable à la conservation, la valorisation et la diffusion à grande échelle du patrimoine audiovisuel suisse. Depuis sa fondation en 1995, MEMORIAV a soutenu plus de 300 projets de sauvegarde.

Néanmoins, près de vingt-cinq ans après le refus de cet institut national, le patrimoine audiovisuel n'est toujours pas protégé et les coûts de récupération, de sauvegarde, et de conservation n'ont pas baissé, au contraire.

La loi sur le dépôt légal dans le Canton de Vaud a été actualisée en avril 2014 (Loi sur le Patrimoine mobilier et immatériel) dans les articles 29 à 34, définissant le rôle des diverses institutions. Les documents audiovisuels ont été omis, il n'y a donc pas d'obligation de dépôt légal auprès de la Bibliothèque cantonale et universitaire de Lausanne pour ces sources. Au vu de ce qui précède, cet état de fait est regrettable : ces sources doivent être conservées et sauvegardées le mieux possible. Les responsabilités sont claires dans la loi sur le Patrimoine mobilier et immatériel (article 29, alinéa 3 et 5):

${ }^{3}$ Le Conseil d'État est l'autorité compétente pour créer des institutions patrimoniales cantonales et les organiser.

${ }^{5}$ Elles peuvent aussi être organisées sous la forme de fondation de droit public par le biais d'une loi du Grand Conseil.

La responsabilité du patrimoine (dans le cadre de ce travail : audiovisuel) est donc bien donnée au Conseil d'État. Il peut donc choisir ou bien de créer un Institut cantonal audiovisuel sous forme une institution patrimoniale cantonale ou bien de lui donner la forme d'une fondation de droit public.

A la création d'un Institut cantonal de l'audiovisuel s'opposent de nombreuses difficultés :

\section{Difficultés financières}

Après le refus de créer un institut national de l'audiovisuel en 1992, il est difficile de retrouver des équipements techniques obsolètes et cela coûte très cher de les garder en état. Néanmoins, il nous semble que la « récupération » des éléments anciens mérite une attention et un investissement particuliers. Le maintien de standards 
informatisés des documents audiovisuels « nés numériques » mérite aussi une attention soutenue. Néanmoins en utilisant des logiciels libres, on simplifie déjà le travail de migration. La politique d'archivage électronique telle qu'elle sera prochainement présentée dans le Canton de Vaud vise à solidariser et à rendre compatibles toutes les étapes de l'archivage, de l'élaboration au versement des documents aux ACV. En travaillant en amont, en faisant connaître les éléments indispensables à un bon archivage, le temps et les ressources financières seront épargnées.

\section{Difficultés liées au volume}

La masse des productions audiovisuelles pose problème. Le fait qu'elles soient le plus souvent nées numériques n'empêche pas la prolifération des sources et le risque de surdosage (trop d'informations tuent l'information). Du point de vue institutionnel, la nécessité s'impose de tout conserver.

\section{La perte de l'unité de fonds :}

La création d'un centre cantonal de l'audiovisuel coupé des autres centres de conservation du patrimoine culturel cantonal risque d'atomiser les principes de l'archivistique en créant la spécialisation par supports ; cela ne permet plus une vue d'ensemble des inventaires, selon notre expérience vécue lors de notre stage à la Cinémathèque suisse ; la documentation (autre qu'audiovisuelle) se trouverait éventuellement ailleurs, et ne permettrait donc pas de saisir l'entièreté d'un fonds. En établissant une répartition par support, le risque est grand de créer - au sens archivistique- une idée de collection plus que la notion d'un fonds ${ }^{16}$.

\section{Autres scénarios envisageables :}

\section{Archives cantonales vaudoises : pôle audiovisuel}

A défaut d'une création d'un Institut Cantonal de l'Audiovisuel (ICA, semblable à l'INA français) vaudois exigeant énormément de ressources financières et du personnel spécialisé, ce patrimoine doit être conservé dans une institution patrimoniale cantonale. Les articles 39 et 40 de la loi sur le patrimoine mobilier et immatériel du Canton de Vaud prévoient une possibilité de créer un fonds spécialement attribué à la sauvegarde de biens d'importance cantonale. Les Archives cantonales pourraient en bénéficier. Les organes politiques vaudois ont doté ce fonds à la hauteur de 100’000

16 Bureau Canadien des Archivistes, Règles pour la description des documents d'archives, version juillet 2008, Annexe D sous le nom : Collection. (1) Regroupement de DOCUMENTS de toute PROVENANCE, rassemblés intentionnellement en fonction d'une caractéristique commune, sur http://www.cdncouncilarchives.ca/rad/rddacomplete_july2008.pdf, le 4 juin 2018. 
francs par année. Cela est loin de répondre aux exigences des différents services intéressés à la sauvegarde du patrimoine culturel.

Par l'attribution par le Conseil d'État et par le Grand Conseil de cette tâche de sauvegarde des archives audiovisuelles, les Archives cantonales vaudoises seraient ainsi maîtres de leurs fonds et des mandats confiés. Elles ne dépendraient plus forcément de la bonne volonté des autres institutions pour mener à bien leurs propres inventaires. Elles seraient à même de chercher les spécialistes et de créer des contrats d'externalisation auprès de différentes institutions, comme la Cinémathèque suisse, voire la Télévision Suisse Romande, ou des organisations commerciales privées capables de travailler avec les différents supports et les normes archivistiques reconnues (par exemple Vectracom en France).

On pourrait imaginer une répartition que l'on connaît dans d'autres pays, autour d'un noyau cantonal et qui engagerait des partenaires communaux, déjà au bénéfice d'expériences dans le domaine. La Cinémathèque suisse pourrait jouer le rôle d'institution de conservation, selon accord avec les institutions concernées qui garderaient la propriété de leurs archives audiovisuelles.

A la Bibliothèque cantonale et universitaire de Lausanne seraient placés les CD - DVD « commerciaux », par analogie au dépôt légal d'imprimés. Suivant l'article 33 de la loi sur le patrimoine mobilier et immatériel, la BCUL, autre institution patrimoniale cantonale, davantage portée par sa nature de bibliothèque patrimoniale sur la diffusion, pourrait animer avec l'aide des archivistes un portail mettant en ligne divers documents audiovisuels, en créant des liens entre les différents autres services d'archives $(\mathrm{RTS}, \ldots)$ à la manière de la médiathèque du Valais.

Aux ACV seraient placés l'audiovisuel non vendu dans le commerce et les fonds liés à l'écrit institutionnel.

Un tel scénario implique un investissement conséquent et pérenne du canton, au bénéfice des Archives cantonales et de la Bibliothèque cantonale et universitaire de Lausanne.

\section{Délégation de compétences cantonales aux Archives de la Ville}

Comme l'a fait le canton de Neuchâtel avec le Département audiovisuel (DAV) de La Chaux-de-Fonds, une des pistes possibles est de donner une compétence cantonale aux Archives de la Ville de Lausanne. Elles sont à même d'offrir une expertise technique, leur expérience ayant été complétée par leurs années d'exercice. Toutefois, cette action n'ira pas sans un investissement du Canton auprès de la commune.

Toutefois, il nous apparaît que les Archives cantonales sont déjà reconnues dans la loi sur le patrimoine mobilier et immatériel comme une institution patrimoniale cantonale et que cela évite la création d'un nouvel organisme étatique patrimonial ou encore d'une fondation de droit public. Il est nécessaire aussi de rappeler que 
le Canton (par l'intermédiaire du Conseil d'État, et par les votes des députés sur les ressources allouées) est responsable de prime abord de sa mémoire culturelle selon cette même loi.

Du point de vue technique ensuite, les archivistes établissent différents standards permettant d'inventorier les images en mouvement, de garantir leur authenticité. Ils sont à même de traiter une masse de documents, contrairement à d'autres institutions comme les musées ou les bibliothèques portés sur l'unité. En créant un dépôt légal audiovisuel, les archivistes seraient à même d'exiger au préalable des producteurs d'images professionnelles des « capsules xml » comportant un certain nombre de métadonnées essentielles. Cela éviterait la nécessité de récupérer a posteriori des éléments déjà présents, surtout dans l'image numérique telle qu'elle est produite actuellement. Le squelette donné par l'inventaire normé avec les métadonnées et -spécialité vaudoise- indexé permet de créer des liens avec d'autres institutions.

Qu'importe le lieu de stockage, seul l'inventaire dressé par un archiviste permet de créer les liens scientifiques et de garantir une « authenticité » de provenance, d'origine, squelette sur lequel s'appuieraient les liens et « un certificat» de bonne utilisation. Cela permettrait d'éviter le réemploi des images tirées de leur contexte, sans aucune mention et les « fake news ».

De plus, il ne faut pas oublier une collaboration efficace avec la Direction des systèmes d'information du canton de Vaud, qui doit permettre la mise en ligne et la mise en place des serveurs nécessaires à la conservation numérique des documents. Cette question est aussi du ressort du Conseil d'État, l'impulsion nécessaire à une communication entre les différents organismes étatiques dépendant de lui.

La question des ressources en téraoctets est essentielle : devons-nous laisser les documents sur Wiki Commons, donc sur des serveurs extérieurs, pas forcément situés en Suisse, comme l'ont fait récemment les Archives cantonales vaudoises avec les photographies d'Albert Naef, premier archéologue cantonal, à l'occasion de la journée internationale des archives du 9 juin $2018{ }^{17}$ ? Devons-nous rester maîtres de notre patrimoine ? Il s'agira d'être extrêmement circonspect quand il s'agira de placer sur cette plateforme des éléments audiovisuels, pour des raisons juridiques et conservatoires.

De plus, la présence d'un site universitaire dans le Canton permettrait d'imaginer une valorisation scientifique des fonds détenus par les différentes institutions

Ces liens entre diverses institutions sont nécessaires, voire indispensables, pour permettre une bonne synergie entre les sources audiovisuelles et leur communication. 
Cela pourrait se faire de manière ponctuelle ou comme nous le proposons de manière pérenne, réfléchie et programmée.

\section{Laisser-faire}

Un dernier scénario qui fait trembler l'archiviste en fin de formation doublé d'un historien serait de laisser s'éteindre faute de moyens et de ressources, voire de réflexion, ce patrimoine essentiel pour la bonne compréhension de notre société. Nous vivons dans un monde paradoxal : nous n'avons jamais produit autant de données, alors que les supports pour les accueillir ne cessent d'être plus fragiles.

\section{Politique publique}

En établissant dans le chapitre 7 de notre travail que le Conseil d'État et le Grand Conseil étaient responsables de ce patrimoine audiovisuel, nous nous remémorons le cours du professeur Nahrath de l'Institut de hautes études en administration publique (IDHEAP de l'Université de Lausanne) reçu durant nos études de Master :

"Une politique publique constitue un enchaînement de décisions ou d'activités, intentionnellement cohérentes, prises par différents acteurs, publics et parfois privés, dont les ressources, les attaches institutionnelles et les intérêts varient, en vue de résoudre de manière ciblée un problème défini politiquement comme collectif.

Cet ensemble de décisions et d'activités donne lieu à des actes formalisés, de nature plus ou moins contraignante, visant à modifier le comportement de groupes sociaux supposés à l'origine du problème collectif à résoudre (groupes cibles), dans l'intérêt de groupes sociaux qui subissent les effets négatifs dudit problème (bénéficiaires finaux) » (Knoepfel, Nahrath, Savary, Varone 2010: 12).

Nous pourrions en conclusion verbaliser ici :

«Si les acteurs politico-administratifs (ici Conseil d'État et Grand Conseil) veulent résoudre le problème public désigné (ici la problématique de la conservation des archives audiovisuelles dans une institution cantonale de patrimoine culturel), alors ils interviennent sur les groupes cibles (Archives cantonales, Bibliothèque cantonale et universitaire, ou un éventuel centre cantonal d'audiovisuel) en vue de modifier leur comportement (conserver ces sources sans savoir qu'en faire) conformément aux objectifs de la politique publique dans l'intérêt des groupes bénéficiaires (chercheurs, usagers des centres, public intéressé) indiqués dans l'identification du problème».

"Si les acteurs politico-administratifs veulent changer le comportement du groupe cible (ici le manque de réflexion du Canton sur leurs archives 
audiovisuelles), alors ils lui imposent de (modalité d'intervention réglementaire), opérer tel ou tel changement de comportement (ici : de déposer leurs documents audiovisuels touchant le Canton dans un centre cantonal de patrimoine culturel) »

En résumé, la sauvegarde du patrimoine audiovisuel vaudois est bien liée à des décisions du Conseil d'État ; il doit donner un cahier des charges précis aux Archives cantonale vaudoises et établir jusqu'où s'étend son périmètre de culture vaudoise. Le Conseil d'État doit être conscient qu'un gros effort (financier) doit être établi à la fois pour le rattrapage des sources anciennes (VHS) et pour l'acquisition de nouveaux formats. Il doit pour cela obliger la direction des services informatiques à se pencher sur la sauvegarde des documents audiovisuels. De plus, une réflexion doit être menée sur la conservation : faut-il placer les documents audiovisuels sur des serveurs avec des sauvegardes ou sur des bandes magnétiques LTO (de moindre coût), avec sauvegardes (ou contrôles ?) obligatoires?

La manière de collaborer entre les différentes institutions en charge du patrimoine vaudois telle que les Archives cantonales vaudoises (dans la conservation) et la Bibliothèque cantonale (dans la diffusion) doit être repensée.

\section{Conclusion}

En regard de toutes les informations recueillies dans ce travail, il en ressort, à titre général, les points suivants :

- Les documents sur support audiovisuel demandent une gestion spécifique et coûteuse tant au niveau de la conservation que de la diffusion.

- La conservation et la communicabilité des données impliquent des transferts sur d'autres supports, soit semblables (analogiques), soit numériques.

- Le débat porte sur la pérennité des supports. On se demande actuellement si la numérisation constitue une solution à long-terme. Partout dans le monde, la numérisation est considérée comme un moyen de sauvegarde, mais qu'en serat-il dans 5 ou 10 ans ?

Au cours de notre réflexion, nous avons, notamment, abordé la problématique de l'acquisition et de l'échantillonnage des données audiovisuelles. Cela a permis de mettre en évidence le rôle indispensable de l'archiviste qui par ses compétences et son expérience peut proposer des solutions pertinentes. Dans ce contexte, les notions de territorialité, d'intelligibilité, de rareté, d'ancienneté et d'authenticité doivent accompagner les professionnels tout au long de leur travail. Il s'agit de ne pas laisser « au hasard informatique » la responsabilité de la sélection.

Aujourd'hui, face à l'accroissement de la volumétrie des données audiovisuelles, nous ne pouvons rester indifférents. Acceptons-nous de laisser disparaître ce 
patrimoine et léguer aux générations suivantes un silence et un trou noir sur le monde vaudois actuel ? Il est urgent de répondre à cette question.

Des pistes sont à étudier sous la direction du Conseil d'État vaudois, autorité légalement compétente pour la création d'institutions patrimoniales : s'agirait-il de créer un Institut cantonal de l'audiovisuel sous forme d'institution patrimoniale ou alors une Fondation de droit public ou encore confier aux Archives cantonales vaudoises une nouvelle mission, celle d'assurer la conservation et la diffusion des documents audiovisuels?

Il serait en effet pertinent que le canton de Vaud se préoccupe de l'archivage des données audiovisuelles institutionnelles, et ce dans un délai raisonnable, soit avant que leur récupération ne soit plus possible. Pour ce faire, les Archives cantonales vaudoises devraient envisager des partenariats avec les institutions patrimoniales possédant déjà des archives photographiques, sonores et filmiques

Si ce travail permet de comprendre la plus-value qu'un archiviste peut apporter en termes de contribution dans l'évaluation des fonds et des collections concernés, il n'en demeure pas moins que la question fondamentale, fil rouge de notre développement réflexif, porte bien sur l'évaluation documentaire du Présent dans une perspective archivistique dynamique pour le Futur, et ce, dans un contexte de politique publique précis. 